\title{
The relationship between leadership and physician well-being: a scoping review
}

This article was published in the following Dove Press journal:

Journal of Healthcare Leadership

31 October 2016

Number of times this article has been viewed

\author{
Anthony J Montgomery \\ Department of Education and Social \\ Policy, University of Macedonia, \\ Thessaloniki, Greece
}

\begin{abstract}
To date, research has established the individual and organizational factors that impair well-being. Thus, we are aware of the organizational "cogs and wheels" that drive well-being, and there is a sense that we can potentially utilize effective leadership to push and pull these in the appropriate directions. However, reviews of leadership in health care point to the lack of academic rigor and difficulty in reaching solid conclusions. Conversely, there is an accepted belief that the most important determinant of the development and maintenance of cultures is current - and future - leadership. Thus, leadership is assumed to be an important element of organizational functioning without the requisite evidence base. Medicine is a unique organizational environment in which the health of physicians may be a significant risk factor for inadequate patient safety and suboptimal care. Globally, physicians are reporting increasing levels of job burnout, especially among younger physicians in training. Not surprisingly, higher levels of physician burnout are associated with suboptimal care for patients and medical error, as well as maladaptive coping strategies among physicians that serve to exacerbate the former. This review is a scoping analysis of the existing literature to address the central question: is there a relationship between organizational leadership and physician well-being? The objectives of the review are as follows: 1) identify the degree to which physician health is under threat; 2) evaluate the evidence linking leadership with physician well-being; 3) identify alternative ways to approach the problem; and 4) outline avenues for future research. Finally, enhancing progress in the field is discussed in the contexts of theory, methodology, and impact.
\end{abstract}

Keywords: leadership, physician well being, burnout, healthcare, quality of care, patient safety

\section{Introduction}

Expecting physicians to deliver safe, efficient, and patient-centered care, while they are getting increasingly burnt out, is not only ineffective but also costly and dangerous. However, despite the reported frequency of physician burnout and the associated consequences on quality of care - and patient safety - burnout is still largely viewed as an individual problem. Currently, all evidence with regard to causal factors associated with physician well-being points to the direction that burnout is an organizational, rather than an individual, problem, rooted in issues related to the working environment and organizational culture. ${ }^{1}$ The style of leadership in a health care organization plays a pivotal role in the experience of physicians. Therefore, this review delineates what the existing literature tells us about the relationship between leadership and physician well-being. The review is structured around answering the following key objectives: 1) identify the degree to which physician health is under threat; 2) evaluate the evidence
Correspondence: Anthony J Montgomery Department of Education and Social Policy, University of Macedonia, Egnatia 156, Thessaloniki 54636, Greece Tel +30 23| 089 I308 Email monty5429@hotmail.com 
linking leadership with physician well-being; 3) identify alternative ways to approach the problem; and 4) outline avenues for future research.

There is relatively little research on the relationship between leadership and well-being generally, and even less among physicians. Therefore, systematic reviews/metaanalyses were not possible in this review. Thus, this review is a scoping review of the existing literature. According to Colquhoun et al, ${ }^{2}$ a scoping review is a form of knowledge synthesis that addresses an exploratory research question aimed at mapping key concepts, types of evidence, and gaps in research related to a defined area or field by systematically searching, selecting, and synthesizing existing knowledge. The overall aim of the review is to answer the question "Is there a relationship between organizational leadership and physician well-being?"

\section{Leadership and well-being: an overview}

To date, research has established the individual and organizational factors that impair well-being. For example, via large epidemiological studies, work characteristics such as job control and support at work, as well as organizational factors such as organizational justice and effort-reward balance, have all been shown to predict health-related outcomes at work (ie, sickness absence and minor psychiatric morbidity). ${ }^{3-5}$ However, knowing the antecedents and outcomes does not translate easily into practical actions. We would expect that appropriate leadership can have an important influence on the factors that influence well-being. To put it simply, we are aware of the organizational "cogs and wheels" that drive well-being, and there is a sense that we can potentially utilize effective leadership to push and pull these in the appropriate directions.

Across the general working population, the evidence base concerning leadership is mixed. For example, in a metaanalysis on the subject, Kuoppala et $\mathrm{al}^{6}$ found only moderate evidence that leadership is associated with job well-being, sick leave, and disability pension (early retirement). Moreover, the evidence was weak regarding leadership and job satisfaction, in addition to being inconclusive regarding job performance. Congruently, in a meta-analysis of leadership impact research, ${ }^{7}$ which specifically focused on leadership interventions, the results were mixed. Overall, there was evidence that interventions had a positive impact, but the effect sizes varied significantly when moderator variables (eg, leadership theory and type of intervention) were assessed. Reflecting on their aggregated data, the authors noted that leadership theories that focused more on behavioral change (ie, participation or performance measures of behavior) may indeed have a greater impact on behavior versus theories focusing on emotional (ie, satisfaction) or cognitive change (ie, level of idea generation). This may reflect the fact that behavioral indicators are more directly linked to organizational objectives. The dependent variables assessed in the review are predominately measured by task and satisfaction with leader performance variables. It is hypothesized that leadership interventions linked to well-being outcomes need a greater focus on emotional and cognitive factors.

The aforementioned studies highlight the fact that leadership research has tended to focus more on organizational and performance-related outcomes and less on the health of the employees per se. Medicine is a unique organizational environment wherein the health of physicians may be a significant risk factor for inadequate patient safety and suboptimal care. ${ }^{8}$

\section{Leadership and medicine}

In terms of medicine, both the Association of American Medical Colleges ${ }^{9}$ and the Accreditation Council for Graduate Medical Education ${ }^{10}$ emphasize the need to develop team leadership and management skills in undergraduate and graduate trainees. Medical schools teach students to become medical leaders, and this fact is reflected in the increasing movement toward including leadership programs at both the undergraduate and postgraduate levels. Given the significant (leadership) role that physicians play in the health care system, we can hypothesize that their impact is greater compared to that of the general workforce. Thus, their clinical training prepares them to take a leading role within the health care system, but such clinical training is not accompanied by the appropriate development of skills needed to lead a team of diverse professionals.

The impact of linking leadership and physician well-being needs to be demonstrated in economic and social terms. The economic cost of ignoring leadership as an important contributor to physician well-being is highlighted by a critical meta-analysis of the literature, which revealed that every dollar devoted to employee wellness programs reduced costs associated with medical leave and absenteeism by approximately $\$ 3.27$ and $\$ 2.73$, respectively. ${ }^{11}$ Health care professionals represent a significant proportion of the workforce in every developed country, and the need to support them will only increase as we go forward into the future. Indeed, in 2008 , it was estimated that $70 \%$ of the health budget in Europe was allocated to salaries and employment-related costs, ${ }^{12}$ while roughly $10 \%$ of the active EU workforce is engaged in the health sector in its widest sense. ${ }^{13}$ In terms of 
the social impact of leadership on physician well-being, there is an opportunity to assess the impact of healthier physicians on patient behaviors in terms of preventive behaviors, adherence to treatment guidelines, and readmission rates. A large US study across seven medical schools provides an important insight into the genesis of professional behavior, whereby cheating/dishonest academic behaviors were rare (reported by $10 \%$ ), but unprofessional conduct related to patient care was more acceptable (reported by up to $43 \%$ ). ${ }^{14}$ It is reasonable to suggest that patient neglect and unprofessional behavior is related to the health of physicians themselves, thus demonstrating that the impact of better leadership on patient outcomes will be a powerful argument going forward.

Before evaluating the evidence concerning the capability of leadership to promote physician wellness, it is appropriate to first review the extent of the problem within health care.

\section{Physicians and their health: the extent of the problem}

Significant numbers of physicians do not take adequate care of their own health. ${ }^{15}$ Thus, it is not surprising that burnout levels among physicians are estimated to be between 25 and $60 \%$ and can reach as high as $75 \% .^{16,17}$ Some studies suggest that physicians are at a higher risk (compared to the general population) for suicide, cardiovascular mortality, and substance abuse. ${ }^{18-20}$

Burnout affects physician performance and well-being, as well as patient well-being. US data indicate that burnout and depression were among the strongest factors related to reporting a recent major medical error among surgeons. ${ }^{21}$ Recent research from seven south European countries, among nurses and physicians, found that burnout was significantly positively associated with higher fast food consumption, infrequent exercise, higher alcohol consumption, and more frequent painkiller use, and these associations remained significant after the inclusion of individual differences and country of residence. $^{22}$ Not surprisingly, risky health behaviors (eg, physical inactivity, alcohol misuse) have been implicated as factors contributing to medical errors and inadequate patient safety, as well as affecting medical professionals' health promotion activities directed toward patients. ${ }^{23-26}$

It is not unreasonable to suggest that there is a symbiotic relationship between physician health and patient outcomes (eg, length of stay, patient satisfaction, and lifestyle changes). Not surprisingly, patient adherence with treatment outcomes is also negatively associated with clinician burnout. ${ }^{27,28} \mathrm{Wal}-$ lace et $\mathrm{al}^{29}$ make a strong case for including physician wellness as a quality indicator for health care systems.
To date, our discussion of well-being is dominated by a pathogenic approach - what has gone wrong - rather than a more holistic approach that seeks to establish what optimal functioning in both the professional and personal domains of physicians looks like. Inadequate work-personal life integration is a significant issue for physicians compared to the general population, ${ }^{30}$ and the notion that younger physicians need to sacrifice their personal/family life for their career will exacerbate burnout and fatigue.

\section{The evidence that leadership can affect well-being}

There is relatively little literature on the topic of leadership and well-being. A further problem arises regarding what actually constitutes well-being and leadership. The definition of well-being is multidimensional and includes reference to happiness, satisfaction, and fulfillment, to name just a few elements. ${ }^{31}$ The evidence that will be reviewed is dominated by an approach to well-being as represented by job burnout. In terms of leadership, there are a myriad of problems associated with defining what it is. ${ }^{32}$ In terms of physicians, there is a difficulty in identifying them as leaders simply based on their location in the health care hierarchy. Moreover, there is an important distinction to be made between the leadership of organizations versus leadership in organizations. ${ }^{33}$ The former relates to what many people consider strategic leadership, while the latter is lower-level face-to-face leadership. When reviewing the literature regarding physicians, it is difficult to ascertain whether physicians are actively engaged in both, and/or whether physicians are considered a leader by their colleagues. A detailed analysis of the epistemological and methodological problems concerning leadership is beyond the scope of this review, but for the purposes of this review, the definition provided by Jaques and Clement ${ }^{34}$ is most appropriate for the context of physicians. According to the authors, leadership is that process in which one person sets the purpose or direction for one or more other persons and gets them to move along together with him or her and with each other in that direction with competence and full commitment. They also stress that leadership is not a free-standing activity: it is one function, among many, that occurs in some, but not all, roles. Both the aforementioned elements match with the work of physicians, particularly in a hospital setting.

Shanafelt et $\mathrm{al}^{35}$ and the Mayo Clinic research team have spearheaded research in this area. For example, a survey of physicians/scientists at a large US health care organization ${ }^{35}$ found that leadership correlated moderately with burnout and strongly with satisfaction (11\% and $47 \%$ 
of the variation, respectively). More detailed analysis of the data reveals interesting results, with the leader's own level of burnout and satisfaction being less important. The authors conclude that the process of medical education creates a situation in which an individual who has not been well prepared to lead is thrust into a very challenging leadership situation. Put simply, physicians are not trained to adequately develop their leadership skills but are expected to be leaders. Congruently, there is evidence that leadership itself can be protective against burnout. A strong link has been observed between career fulfillment and the amount of time physicians are able to devote to the work aspects that are most meaningful to them. If this time comprised $20 \%$ or more of professional endeavors, the risk of burnout was cut in half. ${ }^{36}$ Thus, career fit appears to be a driver of physician wellness. This insight should give clinical leaders pause for thought if they wish to optimize the scientific, medical, and training yield of faculty at their institutions/ health care organizations.

Not surprisingly, the evidence within medicine reflects the more general problem of expecting the underlying factors to translate into actions. The factors that contribute to improved well-being and effective leadership are well established, but knowing the antecedents does not provide a blueprint for interventions. For example, as noted by Shanafelt et $\mathrm{al}^{37}$ in a review on the subject of physician wellness, "providing physicians with increased ability to influence their work environment, to participate in organizational decisions that affect medical practice, and to have more control over their schedules are likely to have a substantial positive effect regardless of practice type". The important part of the previous sentence is "likely", thus we know the elements that must be addressed, but the delivery of health care is complex. Changing work practices and physician behavior will succeed if there is a recognition that silos within health care organizations can lead to a focus on self-preservation rather than a common connected vision linked to patient care.

The introduction of trainee doctors into a health care organization is a critical point in terms of changing or reinforcing system-wide behaviors. Unfortunately, supervisor behavior, in terms of mistreatment and/or humiliation, is associated with a greater likelihood of burnout among trainee physicians. ${ }^{38,39}$ The significant literature on the hidden curriculum suggests that the induction period for many young physicians is characterized by a toxic performance culture, whereby adversity is viewed as "character building" and emotional repression is valorized. ${ }^{40-42}$

Younger physicians report higher levels of burnout, compared to their senior colleagues, and they comprise the group that needs appropriate support from their clinical teachers and senior colleagues. The burnout of residents reflects the cynicism they can feel as a result of the "hidden curriculum" that pervades medical education. ${ }^{40}$ The hidden curriculum refers to the way that junior staff members observe inappropriate behaviors against both colleagues and patients, and which are subsequently imbued with these norms while experiencing feelings of detachment. However, efforts to address burnout among residents are revealing. For example, Ripp et $\mathrm{al}^{43}$ attempted to use facilitated discussion groups to reduce burnout among residents. The authors used a randomized controlled trial design and the intervention involved twiceweekly meetings with an external group facilitator. However, the intervention was ineffective, and the reasons identified by the authors are illuminating. The participants reported that the intervention did not really free them from other clinical and educational responsibilities. Indeed, it may have added to their workload. Additionally, the discussion groups were led by a trained external psychotherapist to allow the participants to engage in unfettered discussions. However, the authors wonder whether selecting internal medicine physicians as facilitators may have led to more substantive discussions around work-related problems.

Part of the problem in disentangling the potential of leadership to contribute to physician well-being concerns the way that leadership is represented by proxy variables. For example, Wallace and Lemaire, ${ }^{44}$ in a sample of Canadian physicians, demonstrated the importance of coworker support, both in terms of being directly related to physician well-being as well as buffering the negative effects of work demands. In the discussion section, the authors suggest that "unnecessary stress can be diminished if leadership increases employees' awareness of organizational goals and involves staff in management decisions." However, the authors did not actually assess any of the aforementioned variables (ie, leadership, awareness of organizational goals, and management decisions), and thus coworker support is used as a proxy variable for leadership and organizational culture.

Demmy et $\mathrm{al}^{45}$ examined the factors that contribute to satisfaction among an idiosyncratic sample of recently hired or fired US physicians. The authors examined 14 factors that contribute to physician satisfaction, but it is not clear for the reader how the 14 factors were initially generated. In conclusion, the authors recommend targeting different leadership practices that affect physicians' satisfaction. Again, it is not clear as to whether they are recommending a reactive leadership approach or one that actively appraises the important elements of satisfaction on a continuous basis. 
Sexton et $\mathrm{al}^{46}$ report on the use of leadership walkrounds (WRs) in neonatal intensive care units (NICUs) and found that WR feedback (ie, received feedback about actions taken to reduce risks) was associated with better safety culture and lower burnout. In terms of burnout, the authors suggest that direct caregiver engagement in patient safety activities at a pace and intensity that are manageable may validate frontline expertise and provide meaning to daily work in a way that reduces burnout. A detailed analysis of their study reveals important specific feedback received by the authors. For example, there was substantial variability between NICUs with regard to the item "Does your clinical area use Patient Safety Leadership WR to discuss with senior leaders any issues that could harm patients or undermine the safe delivery of care?", which limits the conclusiveness of the findings. Moreover, the authors benchmarked their study with adult clinics, but in NICUs, there was less direct participation in leadership WRs and less feedback about actions taken to reduce risk. Finally, while WRs are common in the US, the authors provide absolutely no information on the scope or format of their WRs. This is a significant weakness of the reported research.

Many physicians take on managerial roles in health care organizations. Being in a formal leadership role may buffer well-being, as one may experience feelings of autonomy (control) as a leader, which is negatively correlated with burnout. In a longitudinal study (10 years) of emergency physicians, Cydulka and Korte ${ }^{47}$ found that physicians in professional leadership roles of any kind report more satisfaction and less burnout. The authors noted that they were unable to determine whether more satisfied physicians seek these roles or whether seeking leadership roles leads to greater satisfaction. Moreover, the lack of collegial support strongly predicted burnout.

Overall, the evidence is mixed, but there is relatively little research to make firm conclusions in either direction. Future research should be mindful of the conclusions of Rosenman et al ${ }^{48}$ who in a systematic review of training in health care action teams, conclude that determining best practices in leadership training is confounded by variability in leadership definitions, absence of supporting frameworks, and paucity of robust assessments. At the very least, we can hypothesize that appropriate interpersonal leadership should be able to address the core components of burnout; emotional exhaustion, depersonalization, and feelings of reduced personal accomplishment. However, as already noted, the assessment of well-being needs to include positive and negative indicators.

\section{Approaching the topic from an alternative route}

The previous section reveals that there is relatively little evidence to support the hypothesis that better/effective leadership can improve physician well-being. Thus, we should examine nonphysician examples to explore whether some lessons can be "ferried" back to our physicians.

Laschinger and Fida, ${ }^{49}$ in a sample of Canadian nurses, found that the more the leaders were perceived to be authentic, the less likely that nurses were to experience subsequent work-related bullying and burnout and to want to leave their job and profession. The results highlight the important role of authentic leadership as a job resource in preventing negative employee and organizational outcomes. The Job Demands-Resources (JD-R) model is a useful framework to understand demands and resources in the workplace. In essence, the JD-R model proposes that high job demands lead - via burnout - to negative outcomes (the stress process), whereas job resources lead - via work engagement - to positive outcomes (the motivational process). ${ }^{50}$ The model positions leadership as an important resource; and it represents a useful framework to understand the relationship between leadership and well-being, given that leadership has an impact on follower's job strain and affective well-being. ${ }^{51}$ The JD-R model suggests that leaders are supposed to balance the job demands and job resources of their followers in such a way that they remain healthy, motivated, and productive. ${ }^{52}$ Within health care, managing the allocation and the impact of job demands and job resources on physicians can sustain and improve well-being (particularly for young residents).

In terms of successful interventions, Jones et $a 1,{ }^{53}$ in an impressive series of studies, found stress management interventions could be beneficial to both physicians and their patients. They showed a strong relation between a stressful workplace and malpractice risk in both medical departments and hospitals. Moreover, their results showed significant reductions in medication errors and malpractice claims after the introduction of stress management programs in 22 hospitals; by contrast, rates for the 22 hospitals in the control group (matched on bed numbers, frequency of claims, and rural vs urban) remained unchanged. A deeper examination of their stress management intervention reveals that it operated at five levels: 1) the results of preparatory research demonstrating the link between stress and malpractice claims was communicated to senior management in terms of the link between stress and medication error; 2) the most highly stressed managers worked with consultants to change policy and procedure (ie, interdepartmental communication, 
organization, and personnel policies); 3) senior management shared the results of the survey in small conference sessions, with employee feedback encouraged and action plans made, and management communicated to employees their policy that stress needs to be continually assessed and controlled; 4) all employees were shown videos to help them recognize and cope with stress, and employees were instructed in how to develop better nutrition, exercise, and other health habits (to reduce smoking, substance abuse, and caffeine intake), as well as in how to develop relaxation routines for coping with stresses; 5) a comprehensive employee assistance and counseling program was implemented to allow employees and their families to seek support for work-related and personal problems. The aforementioned study is noteworthy in that it represents an exemplar of how reducing stress can have practical outcomes in terms of quality of care. The exhaustive nature of the intervention (ie, involving all stakeholders at multiple levels) strongly suggests that leadership at all levels of the organization needs to be involved in changing culture.

The potential for leadership to affect positively or negatively is mediated by both the implicit and the explicit ways in which leaders communicate the core values, which shapes the behaviors of employees. However, a recent paper by Nielsen and Daniels ${ }^{54}$ presents important data on the impact of transformational leadership. The authors examined transformational leadership among Danish postal workers over 3 years, and their findings suggest that transformational leaders exert their effects in complex social and temporal processes and may increase sickness absenteeism levels among healthy employees over time. Moreover, their results suggest that some employees in groups with transformational leaders may have increased sickness absence rates if they have high levels of presenteeism, meaning that tranformational leaders motivate employees to come to work even when ill and this short-term gain (attendance at work) results in a long-term undesirable organizational outcome (sickness absenteeism). The results concerning presenteeism are particularly relevant for physicians. More generally, the work by Nielsen and Daniels $^{54}$ questions the assumption that transformational leadership is associated with positive organizational outcomes. The aforementioned result fits with the existing literature within medicine concerning the hidden curriculum and the way that young physicians learn that certain dysfunctional behaviors are valorized (eg, working long hours without appropriate breaks as an indicator of "commitment"). ${ }^{55}$ The aforementioned research should make us wary of simply wanting all physician leaders to be armed with an "MBA box of tools" concerning strategy and vision. Alternatively, they should prompt us to appreciate the historical elements and values of medicine that can contribute to a meaningful and authentic leadership approach, namely, medicine as a vocation and the Hippocratic Oath. Attempts at improving health systems should incorporate existing successful elements, as we want to avoid "throwing the baby out with the bathwater". For example, electronic health records are an obvious improvement for a health system, but they should not result in poorer history taking from patients. ${ }^{56}$ Physicians and nurses are incredibly adept at improvising in restricted environments. Anthropological research, whereby physicians are shadowed over a specified time period, reveals a picture of individuals who "work around" problems to find solutions. ${ }^{57}$ While "work around" strategies that involve short-term fixes to problems are not optimal in the long term, the more important point is that leaders need to tap into this existing creativity and commitment.

\section{Concluding remarks}

Paradoxically, on the one hand, reviews of leadership in health care point to the lack of methodologically rigorous studies and the difficulty in reaching solid conclusions. ${ }^{58}$ Conversely, it has been suggested that the most important determinant of the development and maintenance of proactive organizational culture is current and future leadership. ${ }^{59}$ Thus, the need for better leadership is desirable in spite of a lack of an evidence base to guide researchers and practitioners. A related issue concerns the organizational-professional conflict within health care organizations. Physicians who become managers can occupy a hybrid role between manager and clinician, which blurs the boundaries between the two and results in conflict as the two roles are often experienced as oppositional. ${ }^{60,61}$

Recently, West et $\mathrm{al}^{62}$ at the UK Kings Fund, in a critical report on leadership and leadership development in health care, conclude that health care needs to prioritize overall patient care rather than the success of individual components; build a cooperative, integrative leadership culture; enhancing leaders' learning from experience should be a priority; and evidence-based approaches to leadership development in health care are needed to ensure a return on the huge investments made. Fundamentally, they put forward the argument that the UK health service needs a collective leadership approach. According to West et al, ${ }^{62}$ collective leadership is characterized by shared leadership, where there is still a formal hierarchy, but power is more dependent on who has the expertise at each moment. Thus, collective leadership involves leaders working together to nurture a shared 
culture, adopting leadership styles that are consistent across the organization, and cooperating and supporting each other across boundaries within the organization to deliver continually improving, high-quality, and compassionate patient care. The authors argue that collective leadership has the potential to positively influence overall organizational performance, but it is obvious to see the potential application of collective leadership to staff well-being, in the way that it focuses on better staff support and increased autonomy. The idea of a collective leadership approach is a promising one and builds upon the increasing examples of research that demonstrate the potential for increasing participation among staff and patients.

Physicians are under increasing pressure to continuously improve the quality of care in environments that are not naturally designed to contribute positively to either the health of their employees or the recipients of care. Physicians are educated to be clinicians first, and their role as a leader, team member, or manager is secondary. Thus, the majority of physicians have a tendency to view the purpose of a hospital as primarily to support their clinical work. For example, a survey of American Medical Association members indicated that the majority of physicians do not feel responsible for reducing health care costs. ${ }^{63}$ Congruently, there is evidence that a majority of US surgeons attribute medical mistakes to individual-level factors rather than system issues, ${ }^{21} 70 \%$ vs $15 \%$, respectively. Ironically, a strong belief that individuals, not systems, are primarily responsible for errors calls into question the ability of medical leaders to positively influence the well-being of physicians. A mythology of self-reliance and absolute responsibility is anathema to an interdependent collaborative perspective of health care delivery.

Not surprisingly, this disconnect between physicians' training/expectations - with its emphasis on individual performance - and the realities of the need to work with coworkers and patients who have different visions of how the organization (hospital) should operate means that job burnout is inevitable. ${ }^{8}$ It is not unreasonable to suggest that the current organization of health care, in terms of increasing demands and reduced resources, is directly contributing to the deterioration of physician well-being. The aging population consists of an increased number of patients with chronic diseases, who require complex care provided by several health care professionals, meaning that physicians need to be able to manage/work within multidisciplinary teams, which is crucial for the continuity of care and continuous quality improvement. ${ }^{64}$ Thus, medical leaders need to focus on a combination of organizational and individual strategies, as well as eschew narrow individual approaches that reinforce physicians with the mantra that they are personally responsible to build their own resilience against the prevailing conditions.

\section{Going forward}

To ensure progress in the field, researchers need to address the following issues: better theory, diverse methodological approaches, and bringing evidence from outside medicine.

In terms of theory development, our scientific approach to the issue should heed the recommendations of Rosenman et $\mathrm{al}^{48}$ that we need to develop, test, and adopt leadership frameworks and behavioral taxonomies that can support the rigorous development and assessment of team leadership training. In practical terms, we need integrated solutions, not one-off training days, that identify the specific behaviors that leaders need to shape and reinforce. There is a plethora of leadership theories but not enough consideration of how concepts reverberate with the idiosyncratic nature of health care. We may need to look outside of medicine initially. For example, Schaufeli ${ }^{52}$ has developed an engaging leadership scale that is rooted in the Self-Determination Theory (SDT). ${ }^{65}$ SDT is an established theory that proposes that optimal and healthy functioning is dependent on three elements, namely, competence (feeling effective), autonomy (feeling in control), and relatedness (feeling loved and cared for). These three elements are particularly relevant for physicians and link directly to physician behaviors in terms of practice and training. Developing leaders who enable new physicians to feel effective, in control, and cared for is a good place to start.

Our methodological approaches need to go beyond correlational studies that rely predominately on self-report measures. A mixed-method approach that uses shadowing, diaries, and within-subject designs has the potential to reveal a richer picture of how leadership can affect well-being. Job burnout is a symptom of organizational functioning, so interventions need to address the chronic conditions that are systematically and incrementally contributing to feelings of burnout among staff in a health care organization. In terms of benchmarking organizational issues prior to an intervention, the six areas of worklife spelt out by Leiter and Maslach ${ }^{66}$ is a good place to start. According to this approach, organizations can benchmark six key domains: workload, control, reward, community, fairness, and values. These domains, which are evidence based, can direct organizational interventions toward the key factors influencing individual well-being.

Finally, we can import ideas from outside of the health care arena to inform our approach to leadership. 
Increasingly, in modern health care, physicians work in environments in which multidisciplinary teams work in concert. The need for multidisciplinary teams is rooted in the increased specialization within health care professions, which makes it almost impossible for one professional to be able to provide a holistic care approach, and the fact that multidisciplinary teamwork is considered crucial for the continuity of care and continuous quality improvement. ${ }^{64}$ Across diverse occupations outside of medicine, the factor that has been most strongly associated with effective teamwork is psychological safety. ${ }^{67}$ Most recently, Duhigg, in an article in The New York Times, wrote about how Google investigated what makes its own teams effective via its Project Aristotle. ${ }^{68}$ The results of the investigation suggested that "psychological safety" - whereby team members have a shared belief that it is safe to take risks and share a range of ideas without the fear of being humiliated-emerged as crucial. The considerable literature on the hidden curriculum in medicine highlights how a performance-focused culture can damage feelings of safety and promote a defensive approach to learning and career development. ${ }^{40}$ Building a culture of psychological safety in health care teams has the potential to benefit physicians, their colleagues, and patients.

This review is a snapshot of the key issues concerning leadership and physician well-being. The heterogeneous nature of the literature precluded a systematic review. However, the review has identified the key challenges to be addressed in order for the field to progress.

\section{Disclosure}

The author reports no conflicts of interest in this work.

\section{References}

1. Montgomery A, Todorova I, Baban A, Panagopoulou E. Improving quality and safety in the hospital: the link between organizational culture, burnout, and quality of care. Br J Health Psychol. 2013;18(3): 656-662.

2. Colquhoun HL, Levac D, O'Brien KK, et al. Scoping reviews: time for clarity in definition, methods, and reporting. J Clin Epidemiol. 2014;67(12):1291-1294.

3. North F, Syme SL, Feeney A, Head J, Shipley MJ, Marmot MG. Explaining socioeconomic differences in sickness absence: the Whitehall II study. BMJ. 1993;306(6874):361-366.

4. Kivimaki M, Elovainio M, Vahtera J, Ferrie JE. Organisational justice and health of employees: prospective cohort study. Occup Environ Med. 2003;60(1):27-33.

5. Head J, Kivimaki M, Siegrist J, et al. Effort-reward imbalance and relational injustice at work predict sickness absence: the Whitehall II study. J Psychosom Res. 2007;63(4):433-440.

6. Kuoppala J, Lamminpää A, Liira J, Vainio H. Leadership, job well-being, and health effects - a systematic review and a meta-analysis. J Occup Environ Med. 2008;50(8):904-915.
7. Avolio B, Reichard R, Hannah S, Walumbwa F, Chan A. A meta-analytic review of leadership impact research: experimental and quasi-experimental studies. Leadersh Q. 2009;20(5):764-784.

8. Montgomery AJ. The inevitability of physician burnout: implications for interventions. Burn Res. 2014;1(1):50-56.

9. Association of American Medical Colleges. Recommendations for Clinical Skills Curricula for Undergraduate Medical Education. Task Force on the Clinical Skills Education of Medical Students. Washington DC: Association of American Medical Colleges; 2008. Available from: https://www.aamc.org/download/130608/data/clinicalskills_oct09.qxd. pdf. Accessed May 11, 2016.

10. Accreditation Council for Graduate Medical Education, American Board of Emergency Medicine. The Emergency Medicine Milestone Project. 2012. Available from: https://www.acgme.org/Portals/0/PDFs/ Milestones/PediatricEmergencyMedicineMilestones.pdf. Accessed May 11, 2016.

11. Baicker K, Cutler D, Song Z. Workplace wellness programs can generate savings. Health Aff (Millwood). 2010;29(2):304-311.

12. Commission of the European Communities. Green Paper on the European Workforce for Health. Brussels: Commission of the European Communities; 2008. Available from: http://ec.europa.eu/health/ph_systems/ docs/workforce_gp_en.pdf. Accessed September 29, 2016.

13. European Observatory on Health Systems and Policies. Investing in Europe's Health Workforce of Tomorrow: Scope for Innovation and Collaboration: Summary Report of the Three Policy Dialogues. Belgium: Publication of the Centre for Health Services \& Nursing Research, Catholic University Leuven; 2010.

14. Dyrbye L, Massie FS, Eacker A, et al. Relationship between burnout and professional conduct and attitudes among US medical students. JAMA. 2010;304(11):1171-1180.

15. Montgomery AJ, Bradley C, Rochfort A, Panagopoulou E. A review of self-medication in physicians and medical students. Occup Med (Lond). 2011;61(7):490-497.

16. Goehring C, Bouvier GM, Kunzi B, Bovier P. Psychosocial and professional characteristics of burnout in Swiss primary care practitioners: a cross-sectional survey. Swiss Med Wkly. 2005;135(7-8):101-108.

17. Goitein L, Shanafelt TD, Wipf JE, Slatore CG, Back AL. The effects of work-hour limitations on resident well being, patient care, and education in an internal medicine residency program. Arch Intern Med. 2005;165(22):2601-2606.

18. Arnetz BB. Psychosocial challenges facing physicians of today. Soc Sci Med. 2001;52(2):203-213.

19. Baldisseri MR. Imparied healthcare professional. Crit Care Med. 2007;35(2 suppl):106-116.

20. Center C, Davis M, Detre T, et al. Confronting depression and suicide in physicians: a consensus statement. JAMA. 2003;289(23):3161-3166.

21. Shanafelt TD, Balch CM, Bechamps G, et al. Burnout and medical errors among American surgeons. Ann Surg. 2010;251(6):995-1000.

22. Alexandrova-Karamanova A, Todorova I, Montgomery A, et al. Burnout and health behaviors in health professionals from seven European countries. Int Arch Occup Environ Health. 2016;89(7):1059-1075.

23. Bakhshi S, While AE. Health professionals' alcohol-related professional practices and the relationship between their personal alcohol attitudes and behavior and professional practices: a systematic review. Int $J$ Environ Res Public Health. 2014;11(1):218-248.

24. Fie S, Norman IJ, While AE. The relationship between physicians' and nurses' personal physical activity habits and their health-promotion practice: a systematic review. Health Educ J. 2013;72(1):102-119.

25. Zhu DQ, Norman IJ, While AE. The relationship between doctors' and nurses' own weight status and their weight management practices: a systematic review. Obes Rev. 2011;12(6):459-469.

26. Pipe A, Sorensen M, Reid R. Physician smoking status, attitudes toward smoking, and cessation advice to patients: an international survey. Patient Educ Couns. 2009;74(1):118-123.

27. Haas JS, Cook EF, Puopolo AL, Burstin HR, Cleary PD, Brennan TA. Is the professional satisfaction of general internists associated with patient satisfaction? J Gen Intern Med. 2000;15(2):122-128. 
28. Halbesleben JR, Rathert C. Linking physician burnout and patient outcomes: exploring the dyadic relationship between physicians and patients. Health Care Manage Rev. 2008;33(1):29-39.

29. Wallace JE, Lemaire JB, Ghali WA. Physician wellness: a missing quality indicator. Lancet. 2009;374(9702):1714-1721.

30. Shanafelt TD, Boone S, Tan L, et al. Burnout and satisfaction with work-life balance among US physicians relative to the general US population. Arch Intern Med. 2012;172(18):1377-1385.

31. Dodge R, Daly A, Huyton J, Sanders L. The challenge of defining wellbeing. Int J Wellbeing. 2012;2(3):222-235.

32. Bedeian AG, Hunt JG. Academic amnesia and vestigial assumptions of our forefathers. Leadersh Q. 2006;17(2):190-205.

33. Dubin R. Metaphors of leadership: an overview. In: Hunt JG, Larson LL, editors. Crosscurrents in Leadership. Carbondale, IL: Southern Illinois University Press; 1979:225-238.

34. Jaques E, Clement SD. Executive Leadership. Arlington, VA: Cason Hall; 1991

35. Shanafelt TD, Gorringe G, Menaker R, et al. Impact of organizational leadership on physician burnout and satisfaction. Mayo Clin Proc. 2015;90(4):432-440.

36. Shanafelt TD, West CP, Sloan JA, et al. Career fit and burnout among academic faculty. Arch Intern Med. 2009;169(1):990-995.

37. Shanafelt TD, Sloan JA, Habermann TM. The well-being of physicians. Am J Med. 2003;114(6):513-519.

38. Dyrbye LN, Thomas MR, Harper W, et al. The learning environment and medical student burnout: a multicentre study. Med Educ. 2009;43(3):274-282.

39. Cook AF, Arora VM, Rasinski KA, Curlin FA, Yoon JD. The prevalence of medical student mistreatment and its association with burnout. Acad Med. 2014;89(5):749-754.

40. Gaufberg EH, Batalden M, Sands R, Bell SK. The hidden curriculum: what can we learn from third-year medical student narrative reflections? Acad Med. 2010;85(11):1709-1716.

41. Pitkala KH, Mantyranta T. Professional socialization revised: medical students' own conceptions related to adoption of the future physician's role - a qualitative study. Med Teach. 2003;25(2):155-160.

42. Chang, GC. The Hidden Curriculum: Hazing and Professional Identity [Unpublished Masters Thesis]; 2011. Available from: http://search proquest.com/docview/886783152. Accessed 29 September, 2016.

43. Ripp JA, Fallar R, Korenstein D. A randomized controlled trial to decrease job burnout in first-year internal medicine residents using a facilitated discussion group intervention. J Grad Med Educ. 2016;8(2):256-259.

44. Wallace JE, Lemaire J. On physician well being - you'll get by with a little help from your friends. Soc Sci Med. 2007;64(12):2565-2577.

45. Demmy TL, Kivlahan C, Stone TT, Teague L, Sapienza P. Physicians' perceptions of institutional and leadership factors influencing their job satisfaction at one academic medical center. Acad Med. 2002;77(12 pt 1):1235-1240.

46. Sexton JB, Sharek PJ, Thomas EJ, et al. Exposure to leadership walkrounds in neonatal intensive care units is associated with a better patient safety culture and less caregiver burnout. BMJ Qual Saf. 2014;23(10):814-822.

47. Cydulka RK, Korte R. Career satisfaction in emergency medicine: the ABEM longitudinal study of emergency physicians. Ann Emerg Med. 2008;51(6):714-722.

48. Rosenman ED, Shandro JR, Ilgen JS, Harper AL, Fernandez R. Leadership training in health care action teams: a systematic review. Acad Med. 2014;89(9):1295-1306.

49. Laschinger HK, Fida R. New nurses burnout and workplace wellbeing: the influence of authentic leadership and psychological capital. Burn Res. 2014;1(1):19-28.
50. Schaufeli WB, Taris TW. A critical review of the job demands-resources model: implications for improving work and health. In: Bauer G, Hämmig O, editors. Bridging Occupational, Organizational and Public Health: A Transdisciplinary Approach. Dordrecht: Springer; 2014:43-68.

51. Skakon J, Nielsen K, Borg V, Guzman J. Are leader's well-being, behaviors and style associated with the affective well-being of their employees? A systematic review of three decades of research. Work Stress. 2010;24(2):107-139.

52. Schaufeli WB. Engaging leadership in the job demands-resources model. Career Dev Int. 2015;20(5):446-463.

53. Jones JW, Barge BN, Steffy BD, Fay LM, Kunz LK, Wuebker LJ. Stress and medical malpractice: organizational risk assessment and intervention. J Appl Psychol. 1988;73(4):727-735.

54. Nielsen K, Daniels K. The relationship between transformational leadership and follower sickness absence: the role of presenteeism. Work Stress. 2016;30(2):193-208.

55. Karnieli-Miller O, Vu TR, Frankel RM, et al. Which experiences in the hidden curriculum teach students about professionalism? Acad Med. 2011;86(3):369-377.

56. Chi J, Verghese A. Clinical education and the electronic health record: the flipped patient. JAMA. 2014;312(22):2331-2332.

57. Lemaire JB, Wallace JE, Ghali, WA, Sargious P, Zarnke K, Bacchus $\mathrm{CM}$. Exploring the dimensions of the medical teaching unit physician preceptor role. Abstract Book. In: Association for Medical Education in Europe (AMEE) 2014 Annual Conference; August 30 - September 3, 2014. Milan, Italy. 352.

58. Straus SE, Soobiah C, Levinson W. The Impact of Leadership Training Programs on Physicians in Academic Medical Centers: a systematic review. Acad Med. 2013;88(5):710-723.

59. Schneider B, Barbera KM, editors. Oxford Handbook of Organizational Climate and Culture. New York: Oxford University Press; 2014.

60. Kippist L, Fitzgerald A. Organisational professional conflict and hybrid clinician managers: the effects of dual roles in Australian health care organizations. J Health Organ Manag. 2009;23(6):642-655.

61. von Knorring M, Alexanderson K, Eliasson MA. Healthcare managers' construction of the manager role in relation to the medical profession. $J$ Health Organ Manag. 2016;30(3):421-440.

62. West M, Armit K, Lowenthal L, et al. [webpage on the Internet]. London: The King's Fund; 2015. Leadership and leadership development in health care: the evidence base. Available from: http://www.kingsfund. org.uk/publications/leadership-and-leadership-development-healthcare. Accessed April 7, 2015.

63. Tilburt JC, Wynia MK, Sheeler RD, et al. Views of US physicians about controlling health care costs. JAMA. 2013;310(4):380-389.

64. Nancarrow SA, Booth A, Ariss S, Smith T, Enderby P, Roots A. Ten principles of good interdisciplinary team work. Hum Resour Health. 2013;11:19.

65. Ryan RM, Deci EL. An overview of self-determination theory. In: Deci EL, Ryan RM, editors. Handbook of Self-Determination Research. Rochester, NY: University of Rochester Press; 2002:3-33.

66. Leiter MP, Maslach C. Areas of worklife: a structured approach to organizational predictors of job burnout. In: Perrewe PL, Ganster DC, editors. Research in Occupational Stress and Well-being. Vol. 3. Oxford: Elsevier; 2004:91-134.

67. Edmondson AC, Lei Z. Psychological safety: the history, renaissance, and future of an interpersonal construct. Annu Rev Organ Psychol Organ Behav. 2014;1:23-43.

68. Duhigg C [webpage on the Internet]. What Google Learned From Its Quest to Build the Perfect Team. The New York Times; (February 25 2016). Available from: http://www.nytimes.com/2016/02/28/magazine/ what-google-learned-from-its-quest-to-build-the-perfect-team.html. Accessed 29 September, 2016. 
Journal of Healthcare Leadership

Dovepress

\section{Publish your work in this journal}

The Journal of Healthcare Leadership is an international, peer-reviewed, open access journal focusing on leadership for the health profession. The journal is committed to the rapid publication of research focusing on but not limited to:

Healthcare policy and law; Theoretical and practical aspects of healthcare delivery; Interactions between healthcare and society and evidence-based practices;
Interdisciplinary decision-making; Philosophical and ethical issues; Hazard management; Research and opinion for health leadership; Leadership assessment. The manuscript management system is completely online and includes a very quick and fair peer-review system. Visit http://www.dovepress.com/ testimonials.php to read real quotes from published authors.

Submit your manuscript here: http://www.dovepress.com/journal-of-healthcare-leadership-journal 